\title{
Den chronischen Verlauf stoppen
}

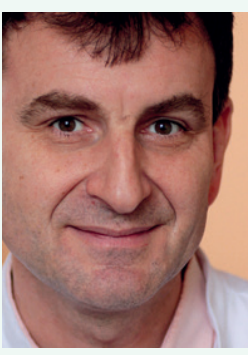

Prof. Dr. med. Christian Grohé ist Chefarzt der Klinik für Pneumologie und Leiter des Lungenkrebszentrums (DKG) der Evangelischen Lungenklinik Berlin. Christian.Grohe@ pgdiakonie.de

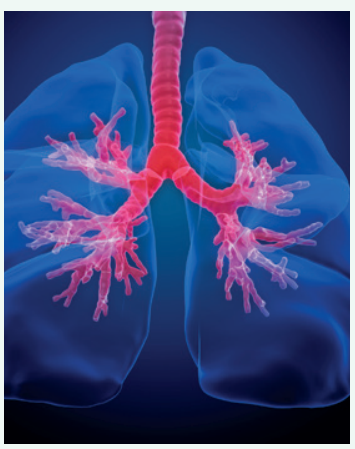

Einer Vielzahl von pneumologischen Krankheitsbildern wie der chronisch-obstruktiven Atemwegserkrankung, aber auch dem Asthma bronchiale liegt eine chronisch-inflammatorische Pathogenese zugrunde. Autoimmunprozesse wie auch die Exazerbationen durch Infekte führen teilweise zu langanhaltenden Krankheitsverläufen. Eine optimale medikamentöse und nichtmedikamentöse Therapie sowie die Schulung der Patienten senken die Komplikationsrate und möglicherweise das Fortschreiten der Komorbiditäten wie z.B. kardiovaskuläre Erkrankungen.

Dem ungenügend kontrollierten chronischen Verlauf ist es geschuldet, dass sich die Anzahl der Patien-

„Die Zahl der Patienten
mit Asthma, COPD und
Bronchiektasien wird
weiter steigen.“

und 2 hinaus, muss ein Spezialist zu Rate gezogen werden. Dieser verfügt über viele medikamentöse Möglichkeiten, die den Krankheitsverlauf unter Kontrolle bringen können. In manchen Fällen zeigen Asthma-Patienten auch Anzeichen einer COPD - besonders wenn eine persistierende Atemwegsobstruktion vorliegt. Handelt es sich dabei um ein diagnostisches Dilemma oder gar um ein neues Syndrom? Prof. Dr. Watz beschreibt in seinem Beitrag die Chancen und Probleme dieses Asthma-COPDOverlap-Syndroms, für das im klinischen Alltag noch keine einheitlichen Therapiekonzepte vorliegen.

Der Beitrag von Dr. Allewelt und Dr. de Roux belegt die Notwendigkeit der detaillierten Abklärung bei persisten mit Asthma, COPD und Bronchiektasien in den letzten Jahren erheblich vergrößert hat und weiter vergrößern wird.

Aktuelle Konzepte zur Behandlung dieser Atemwegserkrankungen sind Gegenstand dieses Dossiers. Zurzeit findet ein spannender Wandel in Bezug auf die individuellen Therapiemöglichkeiten statt. Im Bereich der obstruktiven Atemwegserkrankungen steht die Identifikation der individuellen Belastung des Patienten neben neuen Therapiekonzepten, die die Progression der Erkrankung vermeiden sollen. Dies belegt etwa der Artikel von Prof. Dr. Bergmann: Geht ein Asthma bronchiale über die Schweregrade 1 tierenden oder rasch wiederkehrenden tiefen Atemwegsinfekten - nur so kann einer Bronchiektasien-Entwicklung vorgebeugt werden. Ziel der Therapie ist es, die entzündliche Kaskade zu durchbrechen. Um dies zu erreichen, werden verschiedene Therapieansätze wie die Verordnung von Antibiotika, Makroliden und Physio-

Alle Beiträgen heben ein Ziel hervor: Die zeitgerechte und optimale Behandlung soll den chronischen Verlauf dieser Krankheitsbilder verhindern oder zumindest mindern - und kann so die Prognose der betroffenen Patienten signifikant verbessern. therapie miteinander kombiniert.

\section{Die Artikel im Dossier}

687 Asthma bronchiale - viele Formen, viele Therapien K.-C. Bergmann

694 Non-CF-Bronchiektasen: Aktuelle therapeutische Entwicklungen M. Allewelt, A. de Roux

700 Asthma-COPD-Overlap-Syndrom - Vom diagnostischen Dilemma zum neuen Syndrom? H. Watz 